\title{
3. Microbes and autoimmunity
}

1 ESCHERICHIA COLI EXPANDS THE PROPORTION OF HELIOS ${ }^{+}$NATURAL CD25 ${ }^{+}$CD $127^{\text {LOW/NEG REGULATORY }}$ T CELLS IN NEWBORN CHILDREN

Hardis Rabe, ${ }^{1}$ Anna-Carin Lundell, ${ }^{1}$ Ingegerd Adlerberth, ${ }^{2}$ Agnes E Wold, ${ }^{2}$ Anna Rudin' ${ }^{1}$ Department of Rheumatology and Inflammation Research, The Sahlgrenska Academy at University of Göteborg, Göteborg, Sweden; ${ }^{2}$ Department of Clinical Bacteriology, The Sahlgrenska Academy at University of Göteborg, Göteborg, Sweden 
Background and objectives $\mathrm{CD}^{+}{ }^{+}$regulatory $\mathrm{T}$ cells (Tregs) in human newborns are naïve but fully functional and essential in the maintenance of self-tolerance and immune homeostasis. Naïve Tregs are able to proliferate and still maintain suppressive ability after stimulation. The authors here asked whether bacteria that colonise the gut are able to induce proliferation of naïve Tregs from newborns.

Materials and methods Mononuclear cells were isolated from cord blood of healthy newborns and peripheral blood of healthy adults. The authors defined the regulatory $\mathrm{T}$ cells as the CD4+CD25+CD127low/neg $\mathrm{T}$ cells (Tregs) both in the subsequent cell sorting, the suppression assays and and when subdividing them into helios ${ }^{+}$natural Tregs and peripherally induced helios ${ }^{\text {neg }}$ Tregs. The cell cultures were stimulated with UV-killed bacteria isolated from infants, here Escherichia coli, Staphylococcus aureus or Lactobacilli paracaseii. The authors also analysed the relation between gut bacterial colonisation and regulatory $T$ cells in a prospective newborn-infant cohort of 64 children using multivariate factor analysis.

Results The proportion of Tregs out of CD4 cells was significantly lower in newborns compared to adults and newborns had a significantly higher fraction of helios ${ }^{+}$natural Tregs compared to adults. Tregs from newborns suppressed autologous naïve $\mathrm{CD}^{+}{ }^{+}$responder cells equally well as Tregs from adults. Stimulation with E.coli significantly expanded the proportion of Tregs of $\mathrm{CD}^{+} \mathrm{T}$ cells in newborns in contrast to adults. When subdivided into natural and induced Tregs the authors found that the expansion of the Tregs in newborns was more pronounced in the natural than in the induced Treg fraction. In the prospective newborn-infant cohort the authors found a positive association with early gut colonisation with E.coli but no other gut bacteria with the proportion of cytotoxic $T$ lymphocyte antigen $4\left(\mathrm{CTLA}-4^{+}\right) \mathrm{CD} 4^{+} \mathrm{T}$ cells at 1 month of age.

Conclusions E.coli expands the proportion of preferentially helios ${ }^{+}$natural $\mathrm{CD} 25^{+} \mathrm{CD} 127^{\text {low/neg }}$ regulatory $\mathrm{T}$ cells from newborn children in invitro experiments. These results were supported by the finding that early colonisation with E.coli and no other bacteria was associated to higher proportions of CTLA-4 positive CD4+ T cells in 1-month-old infants in the prospective newborn-infant cohort. Thus, intestinal colonisation with E.coli may play an important role in increasing the proportion of natural Tregs in infants, which may enhance the possibility of maintaining self-tolerance and immune homeostasis. 